\title{
First radioactive beams at ACCULINNA-2 facility and first pro- posed experiment
}

\author{
A.A. Bezbakh ${ }^{1,2,3, \star}$, W. Beekman ${ }^{4}$, V. Chudoba ${ }^{1,3}$, A.S. Fomichev ${ }^{1,5}$, M.S. Golovkov ${ }^{1,5}$, A.V. \\ Gorshkov $^{1,2}$, L.V. Grigorenko ${ }^{1,6,7}$, G. Kaminski ${ }^{1,8}$, S.A. Krupko ${ }^{1,2}$, M. Mentel $^{1,10}$, E.Yu. Nikolskii ${ }^{1,6}$, \\ Yu.L. Parfenova ${ }^{1,9}$, P. Plucinski ${ }^{1,10}$, S.I. Sidorchuk ${ }^{1}$, R.S. Slepnev ${ }^{1}$, P.G. Sharov ${ }^{1,2}$, G.M. Ter- \\ Akopian $^{1,5}$, and B. Zalewski ${ }^{1,8}$ \\ ${ }^{1}$ Flerov Laboratory of Nuclear Reactions, JINR, Dubna RU-141980, Russia; \\ ${ }^{2}$ SSC RF ITEP of NRC "Kurchatov Institute", Moscow RU-117218, Russia; \\ ${ }^{3}$ Institute of Physics, Silesian University in Opava, Bezrucovo nam. 13, Opava 74601, Czech Republic; \\ ${ }^{4}$ SIGMAPHI, F-56000 Vannes, France; \\ ${ }^{5}$ Dubna State University, Dubna RU-141982, Russia; \\ ${ }^{6}$ National Research Center "Kurchatov Institute", Kurchatov sq. 1, RU-123182 Moscow, Russia; \\ ${ }^{7}$ National Research Nuclear University "MEPhl”, Kashirskoye shosse 31, 115409 Moscow, Russia; \\ ${ }^{8}$ Heavy lon Laboratory, University of Warsaw, Pasteura 5a, 02-093 Warsaw, Poland; \\ ${ }^{9}$ Skobeltsyn Institute of Nuclear Physics, Moscow State University, Moscow RU-119991, Russia; \\ ${ }^{10} \mathrm{AGH}$ University of Science and Technology, Faculty of Physics and Applied Computer Science, 30-059 \\ Kraków, Poland;
}

\begin{abstract}
New fragment separator ACCULINNA-2 was installed at the primary beam line of the U-400M cyclotron in 2016. Recently, first radioactive ion beams were obtained. The design parameters of new facility were experimentally confirmed. Intensity, purity and transverse profile of several secondary beams at the final focal plane were studied. The intensities obtained for the secondary beams of ${ }^{14} \mathrm{~B},{ }^{12} \mathrm{Be},{ }^{9,11} \mathrm{Li},{ }^{6,8} \mathrm{He}$ in the fragmentation reaction ${ }^{15} \mathrm{~N}(49.7 \mathrm{AMeV})+\mathrm{Be}(2 \mathrm{~mm})$ are in average 15 times higher in comparison to the ones produced at its forerunner ACCULINNA separator. The ACCULINNA-2 separator will become a backbone facility at the FLNR for the research in the field of light exotic nuclei in the vicinity of the nuclear drip lines. The planned first experiment, aimed for the observation of the ${ }^{7} \mathrm{H}$ nucleus at ACCULINNA-2, is outlined.
\end{abstract}

\section{Present status of ACCULINNA-2}

Since yearly 1990's, intense radioactive ion beams (RIBs) play a crucial role in experiments aimed for study of exotic nuclei lying near and beyond the nucleon drip lines. The fragmentation of incident projectile is proven to be a powerful tool for the RIB production [1]. This technique is employed in a wide range of beam energies (30-1000 AMeV) with the subsequent separation of fragmentation products in-flight. As compared to the known ISOL method used for the production of secondary beams, the in-flight method has two important advantages: (i) typical flight time of fragment from the production target to the final focus of the separator is rather short $(\sim 0.5 \mu$ s for energies of several tens of

^e-mail: bezbakh@jinr.ru 


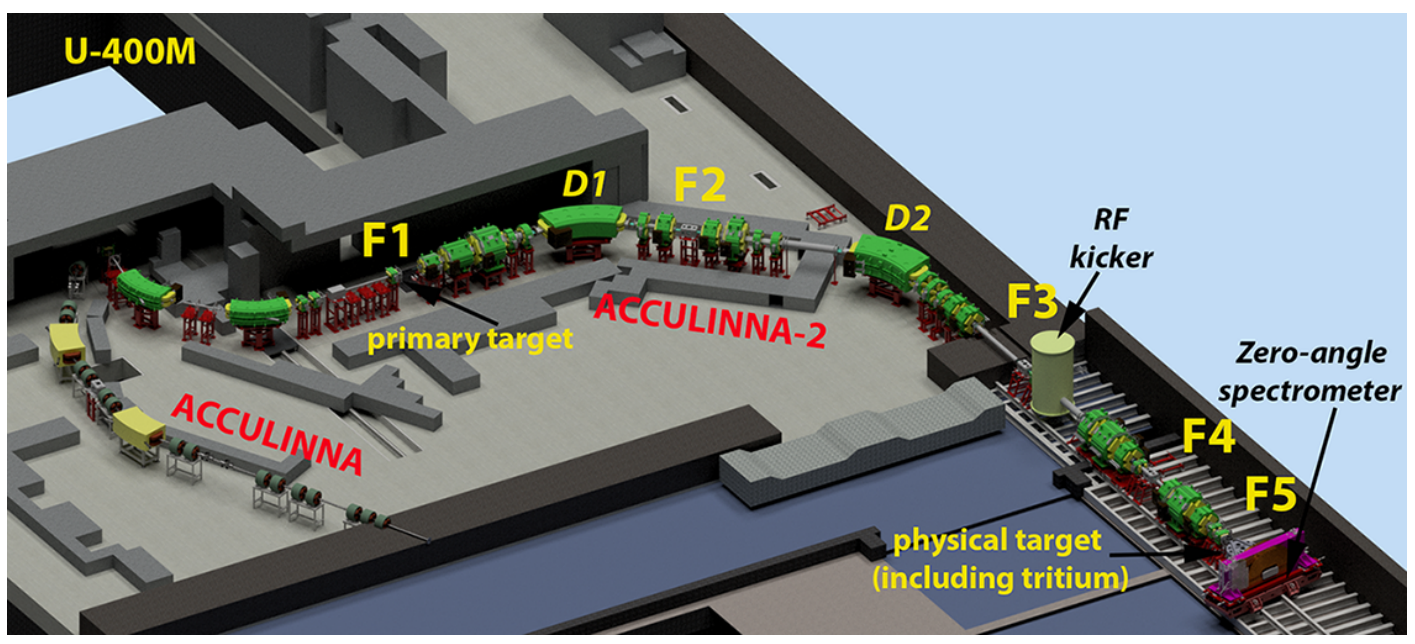

Figure 1. Layout of ACCULINNA-2 fragment separator at U-400M cyclotron. The separator focal planes are indicated by F1-F5, respectively. The main separator part (F1-F3), planed installation of the RF-deflector at F3, and the zero-angle spectrometer at F5 are presented, respectively.

$\mathrm{AMeV}$ ), which means that there is only a smal limitation on the half-life of RIBs;) (ii) fragmentation cross section is large enough to produce with rather high intensities nuclei far from the valley of stability. The ACCULINNA facility was commissioned in 1996 at Flerov Laboratory of Nuclear Reactions, JINR Dubna [2], and upgraded in 2003. High intensity (up to $10 \mathrm{p} \mu \mathrm{A}$ ) primary beams of ${ }^{7} \mathrm{Li},{ }^{11} \mathrm{~B},{ }^{13} \mathrm{C},{ }^{15} \mathrm{~N}$ and ${ }^{18} \mathrm{O}$ with energies between 32 and $50 \mathrm{AMeV}$ were delivered by the U-400M cyclotron to the separator production target. For example, intensity of $6 \times 10^{5} \mathrm{~s}^{-1}$ was reached for the ${ }^{6} \mathrm{He}$ beam with energy of $25 \mathrm{AMeV}$ obtained with a ${ }^{11} \mathrm{~B}$ primary beam at current of $2 \mathrm{p} \mu \mathrm{A}$ hitting the berrylium $\left(350 \mathrm{mg} / \mathrm{cm}^{2}\right.$ ) production target. In 2016, new fragment separator ACCULINNA-2 (see Fig. 1) has been installed at another, extended beam line of the U-400M cyclotron. The separator and a part of the primary beam line were designed and built in collaboration with SIGMAPHI company [3]. It is a achromatic separator consisting of two $45^{\circ}$ dipoles (D1-D2), 14 quadrupoles (Q1-Q14), 5 multipoles (M1-M5) and 2 steering magnets (ST1-ST2). Projectile fragments are produced at focal plane F1 where thick rotating RIB-production target is installed. Secondary ions are separated in flight by using the combination of magnetic analysis and energy losses in achromatic wedge degrader located at the intermediate dispersive focal plane F2. In 2018 a vertically deflecting radio-frequency deflector is planned to be installed at F3 focal plane. The RF-deflector will significantly purify the proton-rich secondary beams. Technical launch of the ACCULINNA-2 facility was carried out in December 2015. The primary beam of ${ }^{32} \mathrm{~S}$ with energy $51.5 \mathrm{AMeV}$ was transported to the $\mathrm{F} 2$ focal plane, and the beam characteristics were examined using a couple of Farraday cups (located at the U-400M output and at F2) and a luminophore located at F2. The beam transmission from U-400M to F1 and from F1 to F2 was found to be more than $90 \%$ and $100 \%$, respectively. These values, together with the size and the beam profile observed in F2, were in accordance with the simulations and fully met the requirements of the project [4]. All commissioning works related to power-supply, water-cooling of the magnetic elements, ventilation and fire-safety were performed during 2016 . The first radioactive ion beams were produced at ACCULINNA-2 in March 2017. The primary beam of ${ }^{15} \mathrm{~N}$ with energy of $49.7 \mathrm{AMeV}$ was delivered to the $\mathrm{F} 1$ focal plane where the $2 \mathrm{~mm}$ thick beryllium 


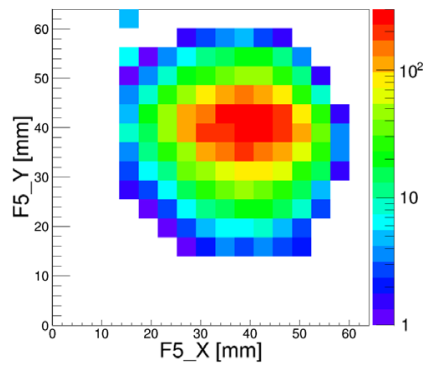

a)

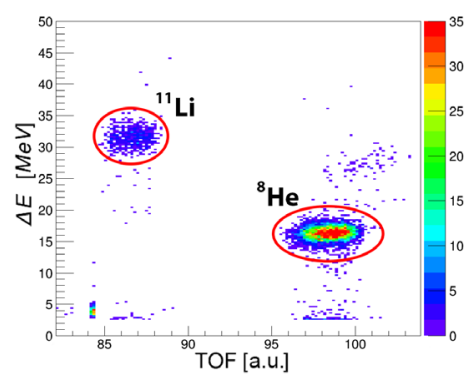

b)

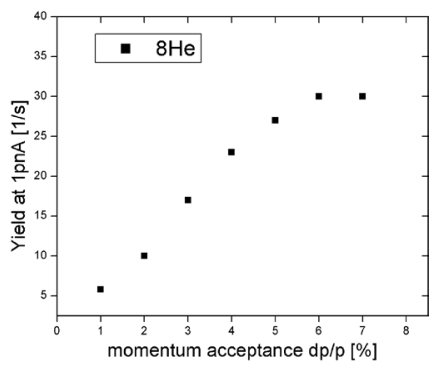

c)

Figure 2. a) Beam spot in the F5 plane obtained by a double-side strip Si detector. The separator elements were tuned for the delivery of ${ }^{8} \mathrm{He}$ to F5. The multipole magnets were swithched off; b) ID plot $\Delta \mathrm{E}$ vs TOF obtained on the $12.3 \mathrm{~m}$ base between F3 - F5 with ACCULINNA-2 tuned on the ${ }^{8} \mathrm{He}$; c) Dependence of the ${ }^{8} \mathrm{He}$ yield on the momentum acceptance of ACCULINNA-2 measured in the focal plane F5. The measurements were performed with $1 \mathrm{~mm}$ beryllium wedge and $\mathrm{dp} / \mathrm{p}= \pm 2 \%$ at $\mathrm{F} 2, \mathrm{Brho(D2})=2.67 \mathrm{Tm}$

production target was installed. The secondary beam was cleaned by means of wedge degrader, made of natural beryllium with thickness of $1 \mathrm{~mm}$, located at F2. The products of fragmentation were registered by the system of RIB diagnostics consisting of a time-of-flight (TOF) and energy losses silicon detectors. The TOF array consisted of a couple of plastic scintillators (BC404) housed in the round-form frames. The thickness of each scintillator was $250 \mu \mathrm{m}$, with diameter of sensitive area of $60 \mathrm{~mm}$. Each scintillator was coupled with four R7600 photomultiplier tubes. The TOF detectors were located at the F3 and F5 focal planes. The distance between both detectors was $12.3 \mathrm{~m}$ and time resolution of TOF system better than $500 \mathrm{ps}$ was established. The $300 \mu \mathrm{m}$ thick double-sided strip silicon detector (Micron-Semiconductor BB7) with sensitive area $64 \times 64 \mathrm{~mm}^{2}$ and with 32 strips on each side was located behind the second TOF detector at F5. This detector served for the measurement of energy losses and beam profile at the final focal plane.

Typical two-dimensional plots presenting the beam spot measured in the F5 final focal plane and the $\triangle \mathrm{E}$-TOF identification pattern obtained when the separator was tuned for the ${ }^{8} \mathrm{He}$ secondary beam are shown in Fig. 2a and Fig. 2b, respectively. Operating with the magnetic multipoles switched off ACCULINNA-2 provides the RIB spots in F5 with typical X/Y dimensions of $28 / 23 \mathrm{~mm}$ as shown in Fig. 2a, while the beam size on the production target was $7 \mathrm{~mm}$ in diameter. The switched on multipoles are important for RIBs with $\mathrm{Z}>3$ to compensate 2-th order aberrations at F2.

Another characteristic for the RIBs production is the dependence of its intensity on momentum acceptance set in the wedge position, i.e. in the F2 focal plane. Such dependence shown for the ${ }^{8} \mathrm{He}$ in Fig. 2c means that the maximum momentum acceptance of ACCULINNA-2 is about $\pm 3 \%$.

The basic parametrs of RIB such as intensity and purity, obtained for a different group of RIBs delivered to the final focal plane F5 of the ACCULINNA-2 separator are presented in Table 1. Intensity of the primary beam was limited to a few pnA in recent measurements because of presence of unprotected from radiation area around the production target (F1) and beam dump (F2). Radiation shielding will be fully completed in 2018 and then the high intensity secondary beams from the U$400 \mathrm{M}$ cyclotron (up to $3 \mu \mathrm{A}$ for ${ }^{15} \mathrm{~N}$ case) will be available. It is worthy to remark that the obtained counting rates (normalized on full primary beam intensity) were in average higher by factor of 15 in comparison with the counting rate of the same RIBs supplied by the ACCULINNA separator. 


\begin{tabular}{c|c|c|c}
\hline RIB & Energy (AMeV) & Intensity $\left(\mathrm{s}^{-1}\right)$ & Purity (\%) \\
\hline${ }^{14} \mathrm{~B}$ & 37.7 & 120 & 65 \\
${ }^{12} \mathrm{Be}$ & 39.4 & 150 & 92 \\
${ }^{11} \mathrm{Li}$ & 37.0 & 4 & 67 \\
${ }^{9} \mathrm{Li}$ & 33.1 & 1100 & 50 \\
${ }^{8} \mathrm{He}$ & 35.8 & 25 & 89 \\
${ }^{6} \mathrm{He}$ & 31.5 & 2700 & 46
\end{tabular}

Table 1. Production rates and purity values for several light neutron-rich RIBs obtained in focal plane F5 when a $49.7 \mathrm{AMeV}$ primary beam ${ }^{15} \mathrm{~N}$, having intensity $1 \mathrm{pnA}$, bombarded a $2 \mathrm{~mm}$ beryllium target installed in the $\mathrm{F} 1$ plane. RIB's intensities and purities were obtained with $1 \mathrm{~mm}$ beryllium wedge and $\Delta p / p= \pm 2 \%$ at $\mathrm{F} 2$.

\section{First day experiments at ACCULINNA-2}

The choice of the first experiment at ACCULINNA-2 strongly depends on the current status of radiation protection. The first experiment scheduled for March 2018 assumes the study of elastic scattering of ${ }^{6} \mathrm{He}$ beam on the deuterium target. Our objective is to obtain parameters of the optical potential for this system. In the second half of 2018, radioactive beams with intensities of up to $10^{5}$ particles per second will be available. This will allow us to perform the study of the ${ }^{7} \mathrm{H}$ nucleus produced in the ${ }^{2} \mathrm{H}\left({ }^{8} \mathrm{He},{ }^{3} \mathrm{He}\right){ }^{7} \mathrm{H}$ reaction. Theory predictions find it conceivable that the ${ }^{7} \mathrm{H}$ exotic nucleus, having not only the extraordinary mass-to-charge ratio, could be tested as being the probable candidate for the $4 \mathrm{n}$ radioactive decay. The possibility that a narrow low-lying resonance of ${ }^{7} \mathrm{H}$ can be observed experimentally was suggested by theoreticians long ago [5]. Now, when available are intensive beams of the well bound nucleus ${ }^{8} \mathrm{He}$, having cluster structure consisting of $\alpha$-core enclosed by $4 \mathrm{n}$ halo (skin), assumption that the ${ }^{7} \mathrm{H}(\mathrm{t}+4 \mathrm{n})$ system might be somehow achieved appears to be realistic.

One of the first attempts to observe the $4 \mathrm{n}$ radioactive decay of ${ }^{7} \mathrm{H}$ set an upper limit of $1 \mathrm{~ns}$ for its lifetime [6]. Searches for the ${ }^{7} \mathrm{H}$ ground-state resonance populated in the reactions ${ }^{1} \mathrm{H}\left({ }^{8} \mathrm{He}, 2 \mathrm{p}\right){ }^{7} \mathrm{H}$, [7], ${ }^{11} \mathrm{Be}\left(\pi^{-}, \mathrm{p}^{3} \mathrm{He}\right){ }^{7} \mathrm{H},[8],{ }^{2} \mathrm{H}\left({ }^{8} \mathrm{He},{ }^{3} \mathrm{He}\right){ }^{7} \mathrm{H},[9,10]$ and ${ }^{12} \mathrm{C}\left({ }^{8} \mathrm{He},{ }^{13} \mathrm{~N}\right)$, [11] were undertaken. The most consistent and sensitive experiment was carried out with the ${ }^{8} \mathrm{He}$ beam at energy $42 \mathrm{AMeV}$ [10]. Despite the poor resolution made available in [10] for the searched resonance state, a peculiarity observed at $\sim 2 \mathrm{MeV}$ in the measured missing-mass spectrum might be understood as indication for some low-energy state (or states) of ${ }^{7} \mathrm{H}$. A cross section of $\sim 30 \mu \mathrm{b} \cdot \mathrm{sr}^{-1}$ was estimated for the low-lying ${ }^{7} \mathrm{H}$ resonance populated in the ${ }^{2} \mathrm{H}\left({ }^{8} \mathrm{He},{ }^{3} \mathrm{He}\right){ }^{7} \mathrm{H}$ reaction.

Meanwhile, the authors of theory work [12] demonstrated recently that in the case of simultaneous emission of two or four neutrons (so-called "true" $2 \mathrm{n}$ or $4 \mathrm{n}$ decays), the extremely long lifetimes are possible even for a fairly large decay energies. The arrangement of experiments aimed for the search for the predicted 4 n-emitters calls for taking serious steps: new methods with a significant improvement in energy resolution and higher beam intensities to achieve large statistics are needed. Among the nearest candidates for the most exotic 4-neutron radioactive decays $\left({ }^{7} \mathrm{H},{ }^{18} \mathrm{Be},{ }^{28} \mathrm{O}\right)$ the ${ }^{7} \mathrm{H}$ system is within realistic reach for the new ACCULINNA-2 facility, and we can join hunting for its discovery.

The reaction ${ }^{2} \mathrm{H}\left({ }^{8} \mathrm{He},{ }^{3} \mathrm{He}\right){ }^{7} \mathrm{H}$ is to be measured at ACCULINNA-2. Considering the large negative $\mathrm{Q}$-value inherent to this reaction the beam energy of about $35 \mathrm{AMeV}$ is optimal to reach the maximum cross sections. The ${ }^{8} \mathrm{He}$ beam intensity, obtainable at ACCULINNA-2, is high enough to keep luminosity at a level of $\sim 2 \times 10^{26} \mathrm{~cm}^{-1} \mathrm{~s}^{-1}$ having the deuterium target thin enough to achieve resolution of $\sim 500 \mathrm{keV}$ in the measured missing-mass spectrum. 


\section{Conclusion}

The new fragment separator ACCULINNA-2 was commissioned at FLNR JINR and the first radioactive beams were obtained. The basic ion-optical characteristics of ACCULINNA-2 described in Letter of Intent [4] have been confirmed experimentally for a number of RIBs. The obtained RIB intensities are by about 15 times larger in comparison with the old ACCULINNA facility. The study of ${ }^{6} \mathrm{He}+{ }^{2} \mathrm{H}$ scattering reaction and the search for the ${ }^{7} \mathrm{H}$ nucleus are planned to be performed at this facility in the near future.

\section{Acknowledgements}

This work was partly supported by RSF 17-12-01367 grant, MEYS Projects (Czech Republic) LTT17003 and LM2015049. The authors express their deep gratitude to FLNR director S.N. Dmitriev and to scientific leader Yu.Ts. Oganessian for the support provided to this work.

\section{References}

[1] J.P. Dufour et al., Nucl. Instr. Methods, A248 (1986) 267.

[2] A.M. Rodin et al., Nucl. Instr. Methods, B126 (1997) 236.

[3] W. Beekman et al., Proc. 13th Intern. Conf. on Heavy Ion Accelerator Technology, p.33, www.nishina.riken.jp/hiat2015.

[4] A.S. Fomichev et al., JINR Communication E13-2008-168, JINR, 2008. http://aculina.jinr.ru/acc-2.php.

[5] Ya.B. Zel'dovich, Sov. Phys. JETP 11, 812 (1960); Zh. Eksp. Teor. Fiz. 381123 (1960).

[6] M.S. Golovkov et al., Phys. Lett. B 588, 163 (2004).

[7] A.A. Korsheninnikov et al., Phys. Rev. Lett. 90, 082501 (2003).

[8] Yu.B. Gurov et al., Phys. Part. Nucl. 40, 558 (2009).

[9] G.M. Ter-Akopian et al., Eur. Phys. J. ST 15061 (2007).

[10] E.Yu. Nikolskii et al., Phys. Rev. C 81, 064606 (2010).

[11] M. Caamaño et al., Phys. Rev. C 78, 044001 (2008).

[12] L.V. Grigorenko et al., Phys. Rev. C 84, 021303(R) (2011). 